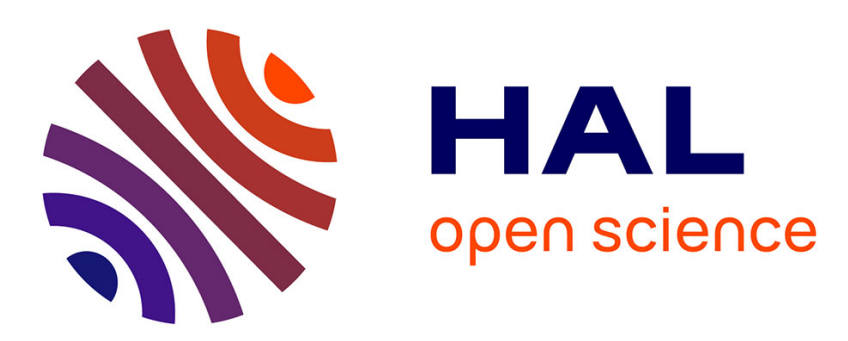

\title{
On the solid-state dewetting of polycrystalline thin films: Capillary versus grain growth approach
}

P. Jacquet, Renaud Podor, J. Ravaux, J. Lautru, J. Teisseire, I. Gozhyk, J. Jupille, R. Lazzari

\section{- To cite this version:}

P. Jacquet, Renaud Podor, J. Ravaux, J. Lautru, J. Teisseire, et al.. On the solid-state dewetting of polycrystalline thin films: Capillary versus grain growth approach. Acta Materialia, 2018, 143, pp.281-290. 10.1016/j.actamat.2017.08.070 . hal-01996081

\section{HAL Id: hal-01996081 \\ https://hal.umontpellier.fr/hal-01996081}

Submitted on 2 Sep 2020

HAL is a multi-disciplinary open access archive for the deposit and dissemination of scientific research documents, whether they are published or not. The documents may come from teaching and research institutions in France or abroad, or from public or private research centers.
L'archive ouverte pluridisciplinaire HAL, est destinée au dépôt et à la diffusion de documents scientifiques de niveau recherche, publiés ou non, émanant des établissements d'enseignement et de recherche français ou étrangers, des laboratoires publics ou privés. 


\title{
On the solid-state dewetting of polycrystalline thin films: capillary versus grain growth approach
}

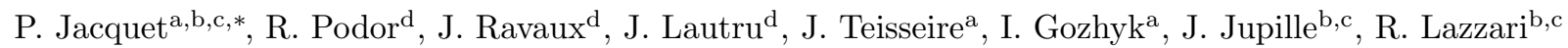 \\ ${ }^{a}$ Surface du Verre et Interfaces, UMR 125 CNRS/Saint-Gobain Recherche, 39 quai Lucien Lefranc, F-93303 Aubervilliers, France \\ ${ }^{b}$ CNRS, UMR 7588, Institut des NanoSciences de Paris, 4 place Jussieu, F-75005 Paris, France \\ ${ }^{c}$ Sorbonne Universités, UPMC Univ Paris 06, UMR 7588, Institut des NanoSciences de Paris, 4 place Jussieu, F-75005 Paris, France \\ ${ }^{d}$ Institut de Chimie Séparative de Marcoule, UMR 5257 CEA-CNRS-UM-ENSCM, Site de Marcoule, F-30207 Bagnols sur Cèze, France
}

\begin{abstract}
Solid-state dewetting of polycrystalline silver thin films was investigated with in situ and real time Environmental Scanning Electron Microscopy at High Temperature (HT-ESEM) in different annealing atmospheres: secondary vacuum or oxygen-rich (partial pressure $\geq 100 \mathrm{~Pa}$ ) environment. A model where oxygen plays a key role is proposed to explain the very different observed morphologies; oxygen favours hole creation and isotropic hole propagation as well as grain selection. But, whatever the atmosphere, dewetting does not proceed through the propagation of a rim but instead involves the growth of specific grains and shrinkage of others. Models based on macroscopic curvature to account for the propagation speed of the dewetting front fail to fit the present observations. This points to a paramount role of the grain size and stability in the dewetting morphology.
\end{abstract}

Keywords: Solid-state dewetting, annealing atmosphere, surface diffusion, in situ Scanning Electron Microscopy, thin films

\section{Introduction}

During the last few years, an increasing research effort has been dedicated to the solid-state dewetting of ${ }_{30}$ metallic thin films 34, 20. It is identified as a poten5 tial way to produce at will metallic structures for numerous applications among which photovoltaic systems or sensors 32] or for low-cost templated fabrication processes at the nanoscale [5]. However, for each application, the ${ }_{35}$ control of the morphology obtained through dewetting is crucial. In this respect, the understanding of the physical phenomena driving dewetting in polycristalline films has been greatly improved. The role of grains has been underlined 13, 2, 15, new diffusion pathways have been identified [17, 16] and the role of crystalline orientation in anisotropic materials has been explored [36. New approaches have been employed and developed to monitor dewetting morphology in situ (Atomic Force Microscopy, AFM [16]) and in real time (Scanning Electron Microscopy, SEM [13, Spectroscopic Ellipsometry, SE 12, Transmis20 sion Electron Microscopy, TEM [26, 25]). These techniques have brought valuable information about the importance of grains in the kinetics of dewetting and the evolution of metallic structures and of holes.

Nevertheless, a consensus in the description of solidstate dewetting has not been reached. Historically, the first model was based on capillary approaches, inspired by

\footnotetext{
${ }^{*}$ Corresponding author
}

liquid dewetting [6], which neglect the crystalline nature of the film. The surfaces are considered as homogeneous and isotropic; the local curvature and the associated gradient of chemical potential is suggested to be the driving force of the material transport. The models imply the propagation of a rim at the dewetting front 6]. However, Jiran and Thompson 14] observed that the rim was not homogeneous, and that the dewetting rate was strongly dependent on its size. The smaller the rim, the faster the propagation. In order to address the specificity of solid surfaces (i.e. anisotropy and faceting), the concept of meancurvature 36] was introduced in the models. Of particular relevance for single crystals [36, 3, it was also used for polycrystalline films [15, 9]. However, in the latter case, the grains are shown to play a non-negligible role [13, 15] leading to a very different approach in which the phenomena involved in the dewetting are related to grain assembly evolution. For instance, the propagation of a rim becomes the successive growth and shrinkage of grains [4]. Instead of a continuous surface, grains are considered as discrete entities exchanging material through specific surface or interface mass transport [16. Here, the chemical potential is directly linked to the size of the grain.

Yet the diversity of studied systems is a great source of complexity in this field of research [34, 20]. From crystalline to amorphous substrates, from films in epitaxy on single-crystals to polycrystalline deposit, dewetting can exhibit numerous morphologies that strongly depend on the 55 system. In the case of films grown on single crystals, holes 
can show geometrical and fractal features constrained by 110 surface energy anisotropy. However, shapes are far less regular upon dewetting of polycrystalline films, in particular on amorphous substrates. In this regard, it is difficult 60 to set up a unique model that applies to any system. For instance, by comparing the dewetting of two different poly-115 crystalline metallic layers, copper and gold [19, Kvon et al. have suggested that two very different mechanisms are responsible for the hole creation within the films. They assign this to relative difference between the metal surface energy and the grain boundary (GB) energy. In the case $e_{120}$ of gold, a morphology of type "nucleation and growth" is observed and was explained with a Diffusion Limited Aggregation model (DLA) while the concomitant appearance of many holes in the $\mathrm{Cu}$ film is attributed to the grooving of grain boundaries.

In the present work, the question of variability of the dewetting morphology is tackled by considering the same system in different annealing atmospheres. The case of

75 polycrystalline silver layers deposited on amorphous silica is examined. This substrate has been chosen to prevent ${ }_{130}$ the behaviour of the film from being dictated by preferential crystalline orientation on the substrate. Dewetting was studied thanks to in situ and real time SEM [13, in different annealing atmospheres, i.e., in the secondary vacuum of the SEM chamber or under near ambient pres-135 sure of oxygen (typically 100-400 Pa). The very different observed morphologies are tentatively explained by a simple model and the role of local curvature in the speed of the propagation front is examined through accurate image analysis.

\section{Experimental and image analysis}

Silver films were deposited by magnetron sputtering (Ar pressure: $8.10^{-3}$ mbar, power: $0.35 \mathrm{~W} / \mathrm{cm}$, rate: $1.3 \mathrm{nnkt5} / \mathrm{s}$ 90 target/sample distance: $8 \mathrm{~cm}$ ) onto polished (100) silicon wafers covered by their native oxides. Wafers were used as received without specific treatment. The thickness of the film (from 15 to $80 \mathrm{~nm}$ ) was controlled by AFM on a step created on purpose in the layer. Post mortem AFM pic- ${ }^{150}$ were acquired on a AFM Dimension Icon microscope (Bruker). All data were recorded at room temperature after the sample had cooled down. SEM experiments were performed with a FEI Quanta 200 Environmental SEM FEG (Field Effect Gun) apparatus in a controlled atmo- ${ }^{155}$ A dedicated in situ heating stage allowed controlling very accurately the sample temperature (between $25-600^{\circ} \mathrm{C}$ ) through a thermocouple placed in direct contact with to the sample 28 . The residual pressure in the chamber was about $10^{-3} \mathrm{~Pa}$ and it will be referred to as ${ }^{160}$

105 "vacuum" condition hereafter. High purity oxygen could also be introduced in the chamber up to a partial pressure of $400 \mathrm{~Pa}$. This environment will be referred to as "oxygen". Images were recorded either at high magnification (typically $\times 10000)$ to study local details of the dewetting layer and to determine the local curvature, or at low magnification $(\times 3000)$ to analyse statistical evolution. When compared, image sequences were recorded from parts of the same initial silicon wafer, ensuring the layer was rigorously the same. Dewetting in vacuum was also studied by TEM on films deposited onto electron transparent amorphous silicon nitride grids. A Tecnai F20 apparatus was run in imaging mode at $200 \mathrm{keV}$, coupled with Automatic Crystal Orientation Mapping (ACOM). ACOM consists in an analysis, pixel by pixel, of the electron diffraction pattern. The local crystalline orientation is reconstructed by fitting the obtained patterned with that calculated from a tilted model crystal. The pixel size $(\approx 5 \mathrm{~nm})$ is larger than that of the probe footprint, which is identical to the TEM in imaging mode, $<1 \mathrm{~nm}$. Data analysis was performed with the Astar-package [30. Due to acquisition time and stability constraints of mapping, experiments were performed at room temperature, after annealing, also preventing further dewetting and change during acquisition. Moreover, the electron beam was directed off the analysed zone during annealing to prevent it from perturbing the dewetting process. Despite the use of different amorphous substrates and imaging techniques, very similar morphologies were found between SEM and TEM runs, further confirming the robustness of the findings.

Specific image processing strategies were developed by using Scikit-Image and Numpy libraries in Python. The local curvature of the dewetting front and its speed of propagation were extracted from the SEM image sequences as follows. First, a segmentation as described in a previous paper [13] is applied to two consecutive images $n$ and $n+1$. The contours of the segregated regions are then extracted with the built-in function skimage.measure.find_contours and fitted with a Spline method (function scipy.interpolate), allowing to calculate easily the local in-plane curvature $\kappa_{\|}$ at each interpolated point. The resulting normal vector at each position of the front in image $n$ is then propagated until it reaches the contour of the image $n+1$. The obtained distance divided by the delay of acquisition gives the local speed $s$ of the front at a given point of curvature $\kappa_{\|}$. This gives rise to a histogram of occurrence $n\left(\kappa_{\|}, s\right)$. However, some curvatures are far more frequently observed than others, and the results should be normalized for the sake of consistency. To understand this, let's consider a circle growing at a constant rate. Obviously, the number of points necessary to describe it grows linearly with its radius. In the corresponding raw $n\left(\kappa_{\|}, s\right)$ histogram, the points at higher curvature (smaller radius) appear less dense than the others. But the physical observation is that the speed of growth is independent from the curvature. To account for that, it is necessary to normalize by the probability of occurrence of a given curvature $n\left(\kappa_{\|}\right)$and to consider the conditional probability $P\left(s \mid \kappa_{\|}\right)$to observe a given speed knowing the curvature:

$$
P\left(s \mid \kappa_{\|}\right)=n\left(\kappa_{\|}, s\right) / n\left(\kappa_{\|}\right) .
$$

To better interpret the following experimental histograms 
of $P\left(s \mid \kappa_{\|}\right)$, let's consider pedagogical examples as illustrated in Fig. 1. A linear front has a curvature constant and equal to zero. If it propagates at a speed $s=V_{0}$, then $P\left(s \mid \kappa_{\|}\right)$consists in an unique point at coordinates ${ }_{170}\left(\kappa_{\|}=0, s=V_{0}\right)$. A circular hole of radius $r$ expanding at constant speed $s=V_{0}$ has a curvature $\kappa_{\|}=-1 / r$ (this curvature sign convention will be kept hereafter : a circular hole has a negative in-plane curvature). At a given time, the histogram $P\left(s \mid \kappa_{\|}\right)$will be represented by a single point of coordinate $\left(\kappa_{\|}=-1 / r, s=V_{0}\right)$. This point will shift towards $\kappa_{\|}=0$ as the radius will increase and the front will look like a linear front. At last, a propagating finger, represented by a shifting half-disk, has a curvature equal either to $\kappa_{\|}=0$ on its side or $\kappa_{\|}=-1 / r$ at the tip of the finger. For the sake of clarity, the points whose propagation speed is zero will deliberately be excluded hereafter. Consequently, $P\left(s \mid \kappa_{\|}\right)$is represented by a segment with constant curvature $\left(\kappa_{\|}=-1 / r\right)$ but with different speeds from $s=V_{0}$ at the dip to $s=0$ at the base.

With the performed image analysis procedure, continuous contours can be determined below pixel size. The maximum curvature $\left(\kappa_{\|} \sim 20 \mu \mathrm{m}^{-1}\right)$ that can be extracted corresponds to a radius value of $\sim 50 \mathrm{~nm}$, close to the resolution of the SEM imaging.

\section{Results}

\subsection{Low magnification images: statistical description}

Let's first focus on images recorded at low magnifications $(\times 3000)$ for which quantities such as the ratio of covered surface or the hole density were constant with respect to magnification. Movies of the dewetting process (see supplementary material, videos 1 and 2 for annealing in vacuum and oxygen, respectively) show that it strongly depends on the atmosphere. Snapshots of the morphologies after dewetting at $350^{\circ} \mathrm{C}$ either in vacuum or in oxygen are shown in Figs. 2 3 respectively. In vacuum (Fig. 2), holes tend to grow with an apparent fractal shape, but no such shape is visible in oxygen (Fig. 3). While dewetting in vacuum seems to follow a "nucleation and growth" behaviour with a few propagating holes, the oxygen atmosphere favours the prompt formation of many holes that coalesce very quickly.

To substantiate these qualitative observations, images were processed to extract the evolution of the substrate coverage and the hole density (Fig. 4). As reported in our previous work [13], it is possible to identify three different steps in the evolutions: (i) induction, (ii) hole propagation and (iii) sintering of disconnected objects. During induction, the surface exhibits no holes but its roughness evolves. In Fig. 4, the onset of hole propagation which is set at the time axis origin corresponds to the beginning of the decrease of the substrate coverage; induction does not appear on the graph. During propagation, the hole density first increases (holes appear), and then decreases (holes coalesce). The end of the process associated with
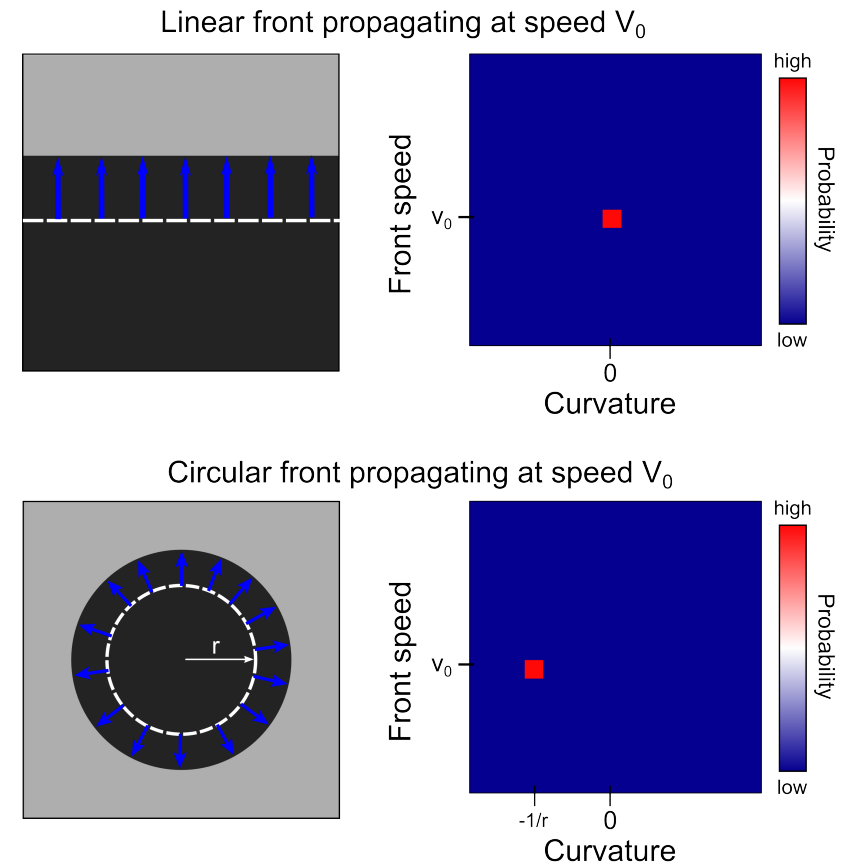

Digit propagating at speed $\mathrm{V}_{0}$
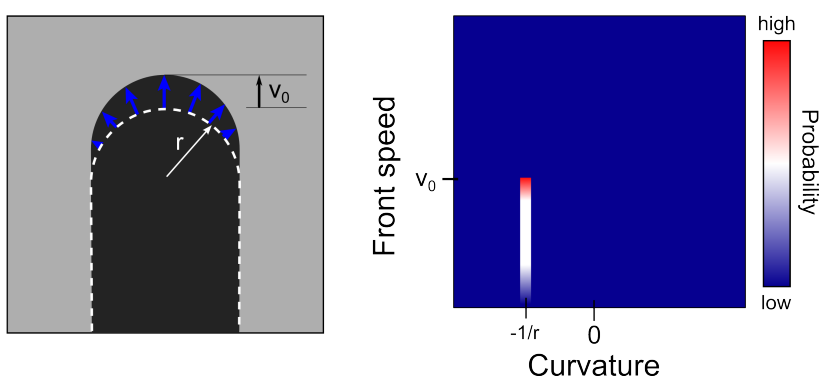

Figure 1: Projected 3D histograms expected of $P\left(s \mid \kappa_{\|}\right)$for three different simple configurations: propagation of a linear front, of a circular hole, or of a finger with circular tip. 

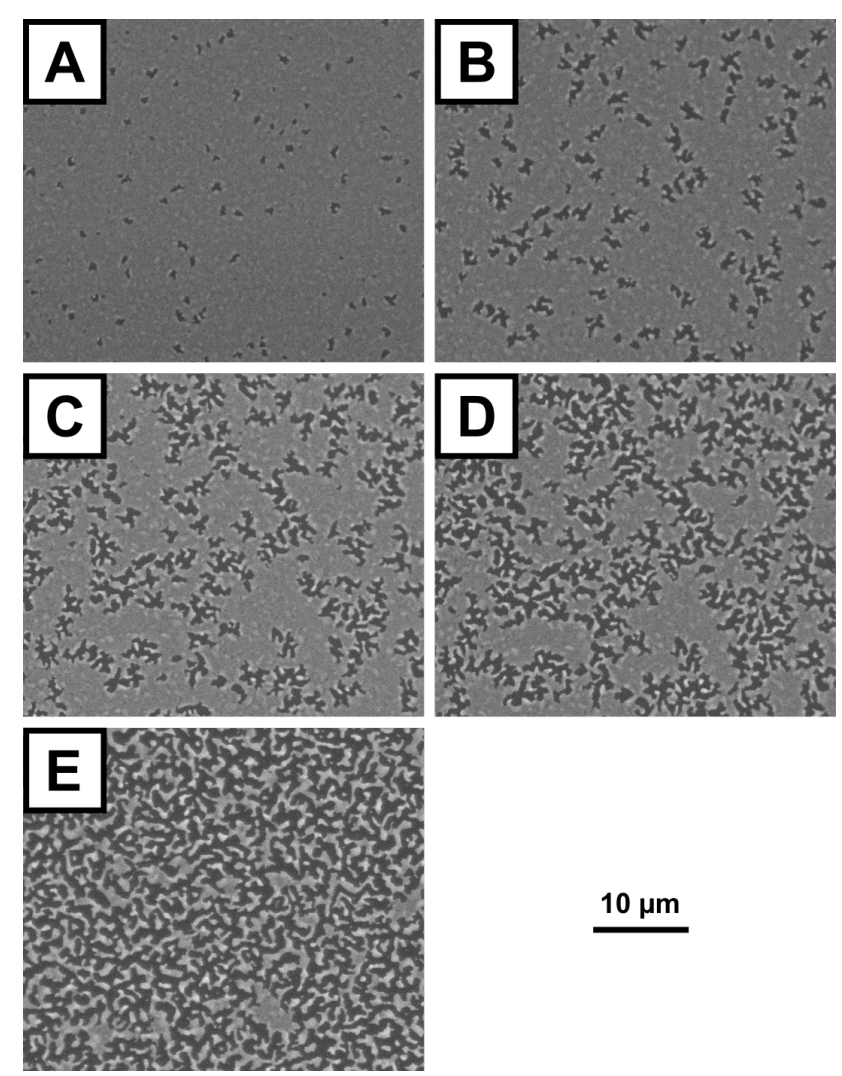

$10 \mu \mathrm{m}$

Figure 2: Snapshots of a $40 \mathrm{~nm}$ silver layer dewetting in vacuum at $350^{\circ} \mathrm{C}$. The delay of acquisition after picture A is respectively 39 , 89,142 and $440 \mathrm{~s}$.
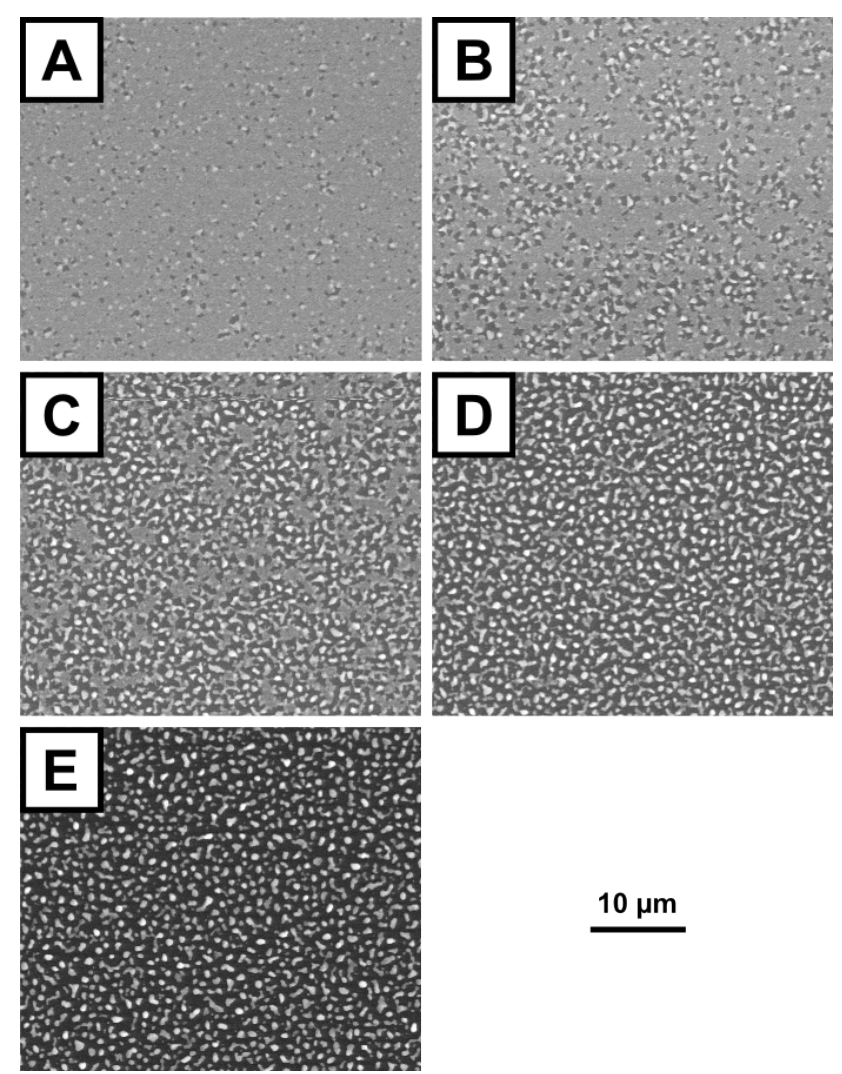

$10 \mu \mathrm{m}$

Figure 3: Snapshots of a $40 \mathrm{~nm}$ silver layer dewetting in a $400 \mathrm{~Pa}$ oxygen atmosphere at $350^{\circ} \mathrm{C}$. The delay of acquisition after picture $\mathrm{A}$ is respectively $14,28,47$ and $1307 \mathrm{~s}$. 


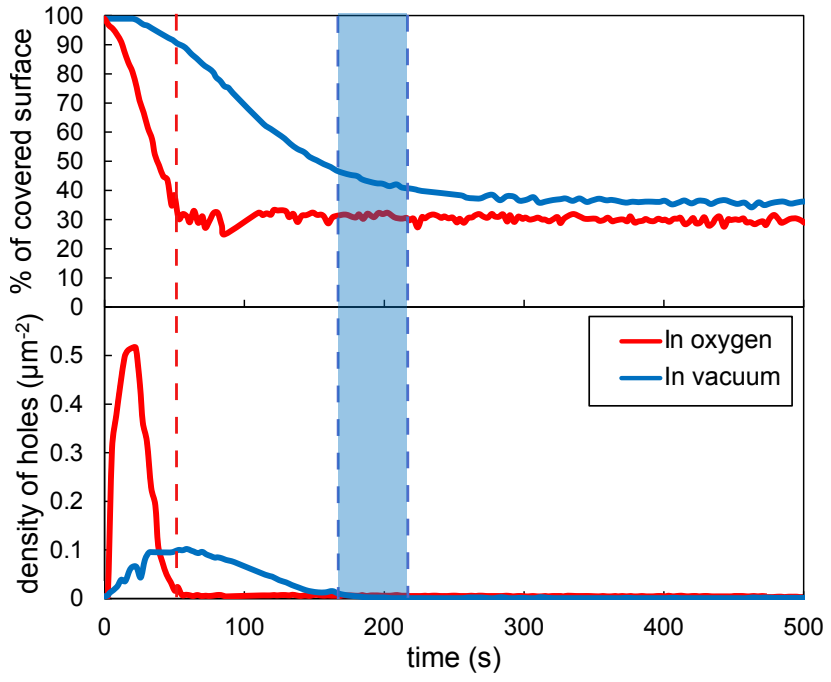

Figure 4: Evolution of substrate coverage by silver (top) and of the hole density (bottom) during the dewetting of a $40 \mathrm{~nm}$ silver layer, in vacuum and in oxygen at $350^{\circ} \mathrm{C}$.

\subsection{High magnification images: morphology of the dewet- ting front}

The local morphology of the dewetting front was then scrutinized at higher magnification (Videos 3 and 4 of supplementary materials, and the associated Figs. 5160). Our 270 previous analysis of dewetting in oxygen atmosphere 13. evidenced that the three steps of induction, hole propagation and sintering are driven by extraordinary grain growth. During induction, some selected grains grow more observed at the end of dewetting. Some grains were observed to grow up to $1 \mu \mathrm{m}$ away from the dewetting front. In contrast to capillary models, no rim was observed during the propagation, because the material was agglomerated 250 into the growing grains, not around the expanding hole (see Fig. 5).

Observations are quite different concerning the dewetting ${ }^{280}$ in vacuum. Instead of a sharp selectivity of grains, several
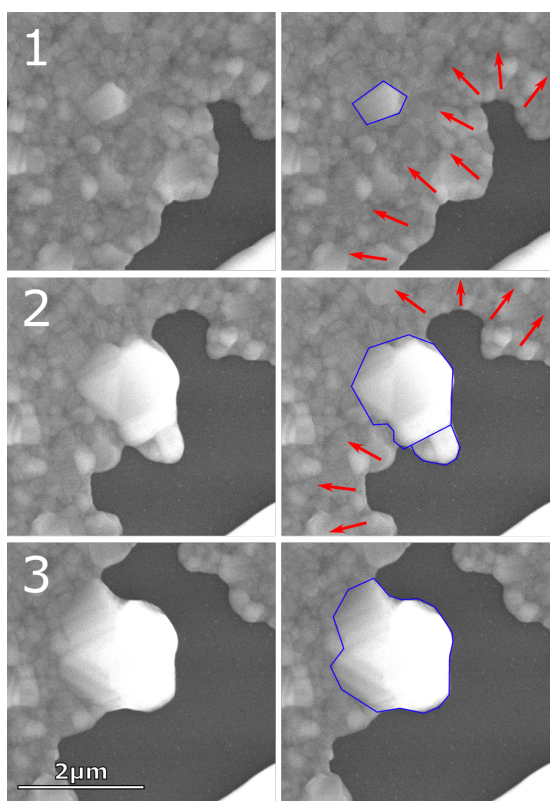

Figure 5: Evolution (left column) of a growing hole in oxygen atmosphere $(400 \mathrm{~Pa})$. Images are recorded during dewetting of a $80 \mathrm{~nm}$ thick silver layer at $480^{\circ} \mathrm{C}$. In the right column of images, the grain contours are highlighted in blue for clarity and the red arrows point to the direction of hole propagation. The delay between picture 1 and 2 is $40 \mathrm{~s}$, the delay between picture 2 and 3 is $1 \mathrm{~min}$ and $40 \mathrm{~s}$.

grains can grow near the expanding holes. The dewetting front propagates in the directions where no material has agglomerated, i.e. where the layer is the thinnest and where no grains have already grown (see Fig. 66). The front takes then a finger shape and bypasses the grains where agglomeration already took place. During propagation, two kinds of zone are typically observed within the silver layer surrounding a hole: (i) zones where the layer remained unchanged since the beginning of the dewetting allowing the dewetting front to progress, and (ii) zones where the silver layer is thicker, because of the agglomeration that took place, preventing the dewetting front from progressing farther. These latter can be assigned to a rim, but this rim is not moving. Consequently and in accordance with the work published by Jiran and Thompson [14, dewetting cannot be described by a propagating rim.

To further assess these observations on the role of oxygen and to rule out beam effects, additional experiments were conducted in a reducing gas mixture of $\mathrm{Ar} / \mathrm{H}_{2}\left(4 \% \mathrm{H}_{2}\right)$ at identical pressure. The observed morphology was very similar to the one seen in vacuum, which means that the presence of oxygen in the annealing atmosphere is indeed the cause of the observed differences.

\subsection{Study of induction}

Our previous AFM study of induction step [13] was performed in air on a $40 \mathrm{~nm}$ thick silver film annealed at temperatures in the range of $100-150^{\circ} \mathrm{C}$. It evidenced a sizeable reorganization of the grains. The film roughening 

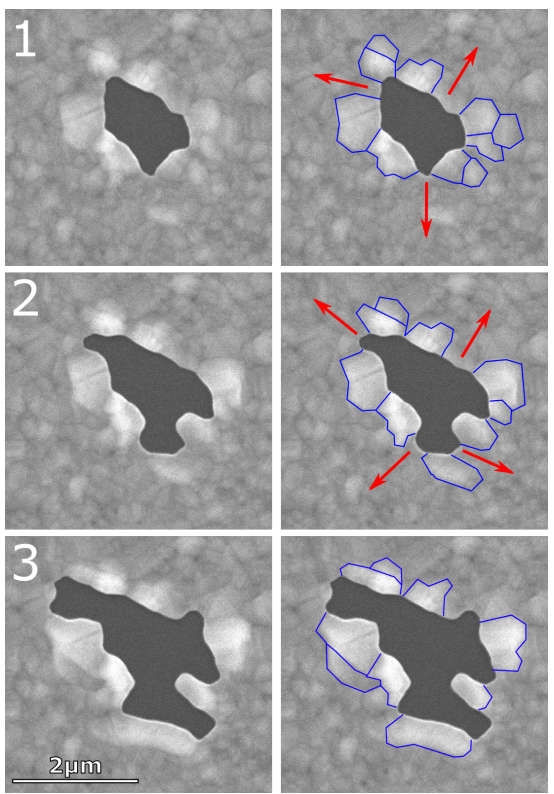

Figure 6: Same as Fig. 5 but in vacuum, annealed at $570^{\circ} \mathrm{C}$. The delay between picture 1 and 2 is $20 \mathrm{~s}$, the delay between picture 2 and 3 is $52 \mathrm{~s}$.

from 3.9 to $4.2 \mathrm{~nm}$ is due to an increase of the out-of-plane grain size, as well as that of the peak to valley amplitude (i.e. top of grains to grain boundaries). At some point, the depth of the valleys equals the thickness of the film, leading to the creation of holes. Since AFM hardly probes facets directions, the possible role of crystalline orientations in the selectivity of grains was approached with TEM. Automated Crystal Orientation Mapping (ACOM) was performed in the vacuum of a TEM column on thinner films $(15 \mathrm{~nm})$ because of the electron transmission requirement. The obtained images (Fig. 7) consists in a color map of the crystallographic axes normal to the surface. Due to obvious crystalline symmetry, the color palette is divided into $\langle 100\rangle,\langle 110\rangle,\langle 111\rangle$ orientations. On the as-deposited film (Fig. $7 \mathrm{a}$ ), the contribution from $\langle 111\rangle$ (green) and $\langle 100\rangle$ (dark blue) orientations dominate while $\langle 110\rangle$ one (red) are scarce. Beyond the question of interface and grain boundary energies, this findings is qualitatively in line which the $e_{320}$ hierarchy of the surface energies $\gamma$ of silver which increase in the order $\gamma(111)<\gamma(100)<\gamma(110)$ [21, 33, with, nevertheless, a low anisotropy. After a moderate annealing at $75^{\circ} \mathrm{C}$ in vacuum (Fig. $7 \mathrm{fb}$ ), sizeable changes are observed but yet the main features are easily recognizable. For in- ${ }_{325}$ stance, some grains grew (as indicated by the red arrow in Fig. 7 a,b), whereas some others split (as shown by the light blue arrow). After annealing at $100^{\circ} \mathrm{C}$ (Fig. 7 fc), the changes are so dramatic that it is not possible any more to identify the outcome of each grain despite an accurate re- ${ }_{330}$ location on the same area after thermal drift of the sample. Even the biggest grains exhibit different out-of-plane orientation. Regarding relative contributions of orientations, the $\langle 111\rangle$ one increased. The initial in-plane mean grain 315 size estimated from ACOM maps evolved from $12 \mathrm{~nm}$ up
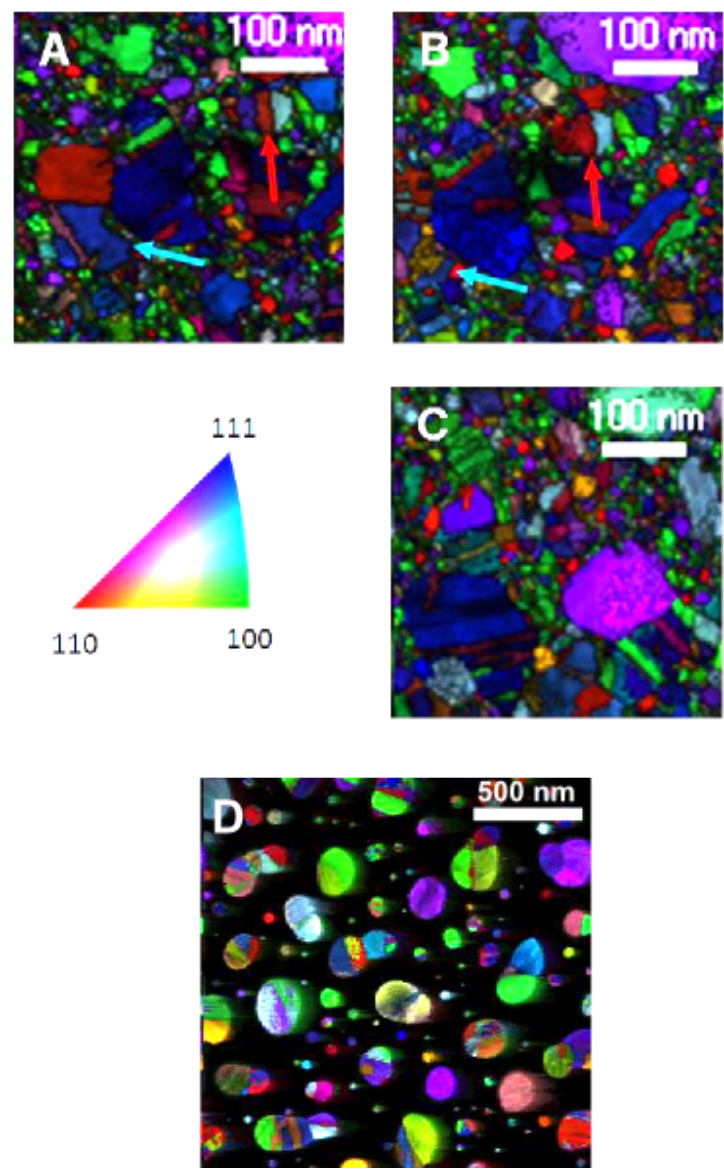

Figure 7: ACOM maps of the out-of-plane orientation $\langle h k l\rangle$ of grains in a $15 \mathrm{~nm}$ silver layer: a) as deposited, b) after annealing at $75^{\circ} \mathrm{C}$, c) at $125^{\circ} \mathrm{C}$ in the $\mathrm{TEM}$ chamber and d) after annealing at $400^{\circ} \mathrm{C}$ in air. Once reached, the temperature of annealing was kept during 5 min before cooling down. The corners of the color code are associated to the three main orientations $\langle 111\rangle,\langle 110\rangle,\langle 100\rangle$ while black zones corresponds to amorphous parts or areas where the orientation identification is ambiguous.

to $23 \mathrm{~nm}$ after the second annealing. To sum up, a significant crystallographic reorganisation and growth of grains is observed during induction.

Due to technical limitations, such ACOM mapping can not be conducted under oxygen atmosphere. Therefore, the orientation of one sample after annealing in air was analysed post mortem. As shown in Fig. 7fd, there is no clear favoured orientation although the $\{100\}$ surface energy is known to dramatically decrease upon oxygen exposure with respect to $\{111\}$ and $\{110\}$ faces [21]. But ACOM, in a similar way to Bragg-Brentano X-ray diffraction, determines only orientations normal to the surface, it may not be sensitive to a favoured growth along a tilted orientation.

\section{Discussion}

Dramatically different morphologies are observed during the annealing of a silver film just by changing the atmo- 
sphere. A parallel can be drawn between our observations those made by Kwon et al. 19 studying copper and gold in reductive atmosphere. Dewetting patterns of gold at $700^{\circ} \mathrm{C}$ are very similar to that of silver in vacuum while dewetting morphology of copper at $300^{\circ} \mathrm{C}$ closely resemble that of silver in oxygen atmosphere. However, advantaly, many physical parameters, such as the initial state of the layer, remain strictly identical in our study; only oxygen is the cause of the observed differences. Oxygen is known to interact with silver in various ways [31, 7, 24]. At high temperatures $\left(700-900^{\circ} \mathrm{C}\right)$, oxygen increases the due to its adsorption on it 35. Oxygen is also known to reduce silver surface energy, in a similar way in liquid 8 or solid state [7, 21. Diffusion enhancement and surface energy decrease are the two main possible explanations of ${ }_{390}$ 350 the influence of oxygen on dewetting morphology.

By extrapolating to our temperature range $\left(300-570^{\circ} \mathrm{C}\right)$ the studies demonstrating the increase of silver self diffusion under oxygen, an enhancement of the diffusion coefficient by two orders of magnitude [31, if not more, is 355 to be expected between our vacuum and oxygen atmospheres. But a close comparison to the work of Kwon et al. 19, rules out diffusion enhancement as the key parameter inducing the difference in dewetting morphology. Indeed, diffusion is faster in the case of gold $\left(\mathrm{D}_{A u}\left(700^{\circ} \mathrm{C}\right)={ }_{40}\right.$ $\left.3604.610^{-6} \mathrm{~cm}^{2} \cdot \mathrm{s}^{-1}\right)$ than in the case of copper $\left(\mathrm{D}_{C u}\left(300^{\circ} \mathrm{C}\right)=\right.$ $7.810^{-7} \mathrm{~cm}^{2} . \mathrm{s}^{-1}$ ) [1. However, in our case, the "copperlike" morphology is observed in presence of oxygen for which the diffusion is assumed to be faster whereas the opposite would be expected. Independently of diffusion, Kwon et al. 19 have suggested that the ratio between the surface and GB interface energy strongly impacts the ${ }^{405}$ resulting morphology, via the grooving of grain boundaries 22. This possible explanation is now explored on both induction and propagation steps.

\subsection{Induction, oxygen and grain boundary grooving}

Induction was already pointed out by Presland et al. 29] in his pioneering work on dewetting of silver on silica. During induction, there is no variation in substrate coverage, yet many transformations occur. Presland et al. observed the formation of hillocks with SEM and suggested that ${ }^{415}$ they resulted from the relaxation of stress arising at the interface due to the difference of thermal expansion between film and substrate. As thin polycrystalline films consist of many grains in contact, our previous results clearly evidence that hillocks are grains that grew selectively. Similar ${ }^{420}$ changes are indeed observed within a polycrystalline layer in vacuum (see Sect. 3.3.)

The dramatic reorganization that occurs during induction, both in vacuum or in oxygen atmospheres leads however to different outcomes (see Fig. 8). After annealing in oxy- ${ }^{42}$ gen, many holes form promptly from an initially increased roughness (density $=1.2 \mu \mathrm{m}^{-2}$ for a $40 \mathrm{~nm}$ thick film annealed at $\left.150^{\circ} \mathrm{C}\right)$. However, after annealing in vacuum, despite the strong reorganization, the layer remains mostly
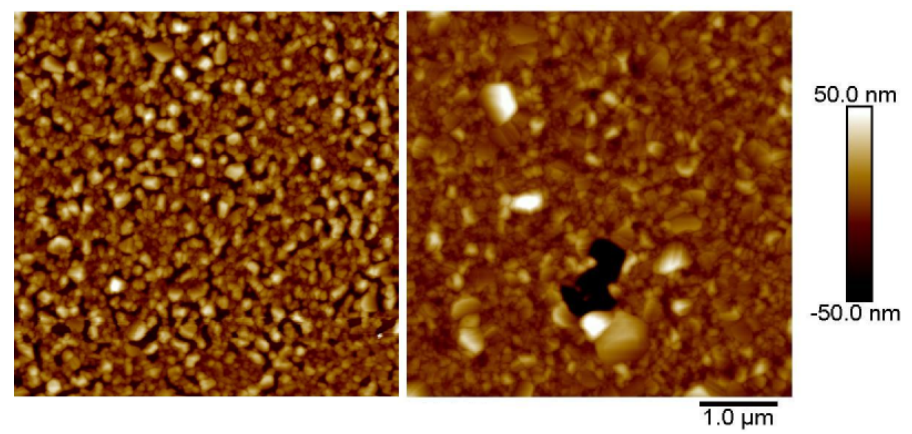

Figure 8: AFM pictures taken on partially dewetted $40 \mathrm{~nm}$ thick silver layers. Left: after annealing at $150^{\circ} \mathrm{C}$ for $5 \mathrm{~min}$ in air. Right: after annealing in vacuum at $300^{\circ} \mathrm{C}$ for $30 \mathrm{~min}$.

continuous with only a few holes appearance (density = $5.10^{-3} \mu \mathrm{m}^{-2}$ for a $40 \mathrm{~nm}$ thick film annealed at an higher temperature of $300^{\circ} \mathrm{C}$ ). Moreover, the grains remaining in the parts of the undewetted layer have a greater in-plane size in the range of a few hundreds nanometers. This AFM observation is in accord with the TEM ACOM findings showing a strong crystallographic reorganization. This latter is still ongoing, but it is not causing the creation of holes as it does in oxygen atmosphere (see Fig. 8-right).

By lowering the silver surface energy [7, 21, oxygen will change the equilibrium state at the triple line between two grain boundaries in contact with the atmosphere. Indeed, the equilibrium at this junction reads:

$$
\gamma_{G B} / \gamma_{M}=2 \cos \left(\theta_{G B} / 2\right)
$$

where $\gamma_{G B}$ and $\gamma_{M}$ are the energies of the grain boundary and of the free surface, respectively and $\theta_{G B}$ the dihedral angle formed by the surfaces of the two touching grains. An estimate of the ratio $\gamma_{G B} / \gamma_{M}$ as the function of oxygen activity can be obtained from energetics data of the literature. We considered the data from Ref. 7 for the silver surface energy versus oxygen activity (although being obtained at higher temperature of $930^{\circ} \mathrm{C}$ ) and we used different estimates of grain boundary energies that we assumed to be insensitive to oxygen [11, 18, 10, to calculate the theoretical angle. The values are plotted in Fig. 9. For each value of $\gamma_{G B}$, a sizeable drop of $\theta_{G B}$ is expected when the oxygen activity increases.

Mullins modelled grooving 23] as a steady profile evolution of the grain boundary down to the interface with the substrate, which apparently does not correspond to our observations. In our case, independently on the pathway of diffusion of silver (at surface or interface), the successive reorganization of grains is the main source for grooving: it happens through the appearance of new contact angles, instead of a continuous transformation. Let us consider the schemes of the Fig. 10 that represent a crystalline structure that is about to evolve (top), either in vacuum or in an oxygen-rich atmosphere. Since the reduction of the 


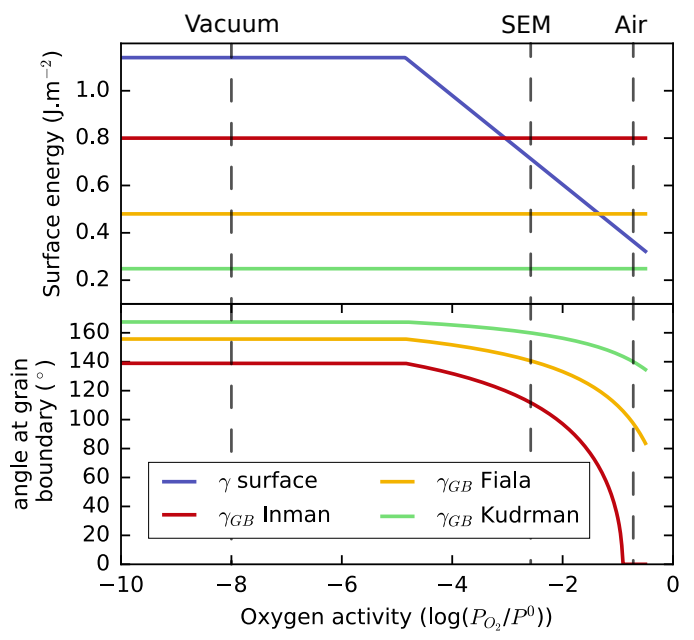

Figure 9: Calculated equilibrium dihedral angle at grain boundaries as function of oxygen partial pressure. Numerical values are taken from Refs. 7, 11, 18, 10]. The dashed vertical lines indicate the conditions used herein.

total energy of the system is the main driving force for grain reorganization, it is logical to assume that it leads to configurations closer to the equilibrium state at the triple line. As explained above, since this equilibrium drastically depends on atmosphere, different morphologies are expected between vacuum and oxygen. This explains the appearance of many holes in oxygen atmosphere : induction favours deep grooves in this atmosphere.

\subsection{Role of local curvature in the propagation}

As mentioned above, the shape of the holes during dewetting is environment-dependent. In absence of oxygen, holes tend to form dendrites and the propagation exhibits a very characteristic fractal morphology, comparable to the gold dewetting in Kwon's work 19. To quantitatively compare data, the dependence of the local propagation speed of the front on the local curvature was extracted as explained in Sect. 2. The results presented below are obtained from videos 5 and 6 (from Supplementary Material), obtained on a $60 \mathrm{~nm}$ thick silver layer, dewetting at $270^{\circ} \mathrm{C}$ in oxygen or at $430^{\circ} \mathrm{C}$ in vacuum, respectively.

As shown in Fig. 11-top, in oxygen atmosphere, the speed of the propagation of the dewetting front does not depend on the local in-plane curvature. In contrast, in vacuum (Fig. 11 bottom), the more negative the in-plane curvature $\kappa_{\|}$, the higher the probability to observe high speeds. This demonstrates that if a dendrite appears, the propagation speed will be faster at its tip than on its side. Surprisingly, the maximum of speed is reached for fingers with a in-plane curvature of around $16 \mu \mathrm{m}^{-1}$ corresponding to a radius of the order of the film thickness $(62.5 \mathrm{~nm})$.

We also notice an increase of this speed at high positive curvatures (around $18 \mu \mathrm{m}^{-1}$ ). This indicates that finger retractation is also observed during the sequence. It is not, however, very representative of the hole propagation.

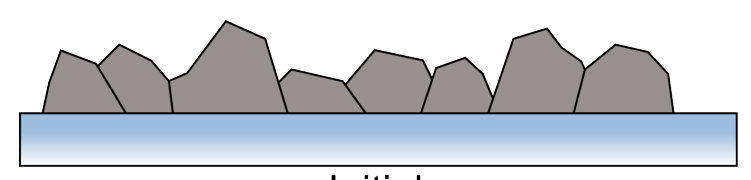

Initial

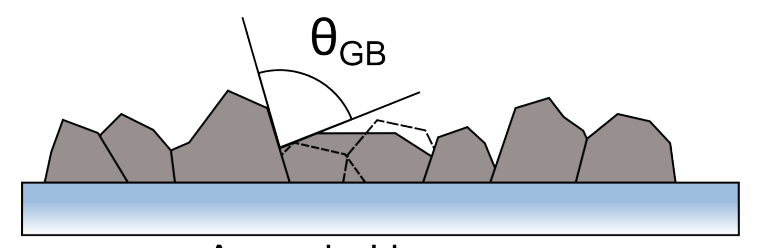

Annealed in vacuum

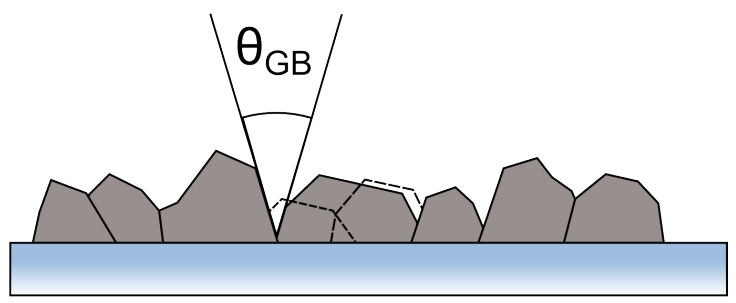

Annealed in oxygen atmosphere

Figure 10: Schematic representation of grain reorganization in different atmospheres.
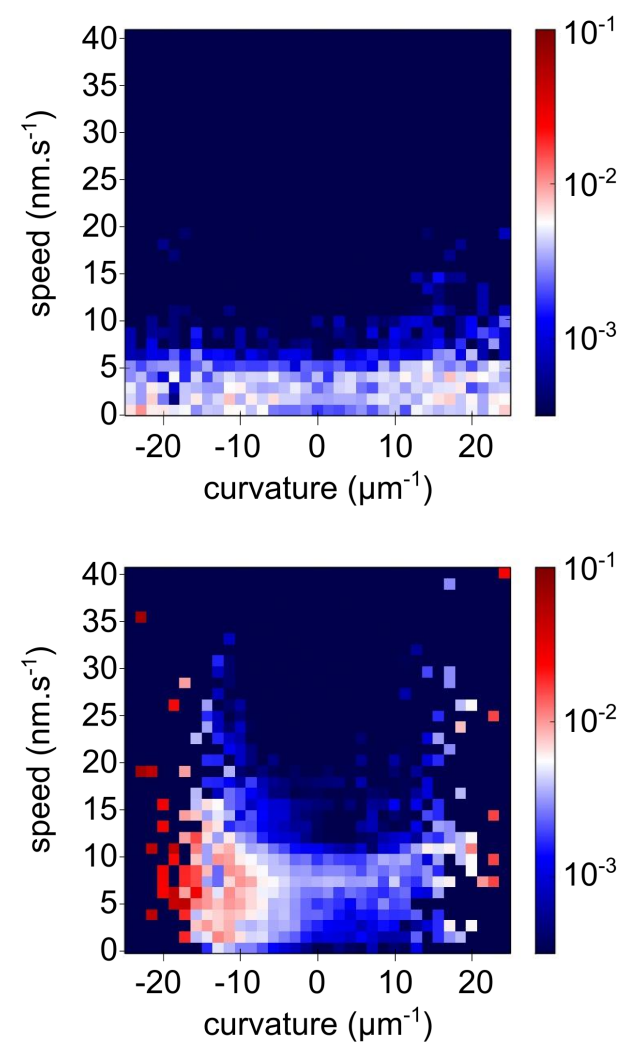

Figure 11: Probability $P\left(s \mid \kappa_{\|}\right)$given the curvature of observing a local speed versus $\kappa_{\|}$(see text for explanations). Top: in oxygen atmosphere. Bottom: in vacuum. Data are extracted the image sequences of videos 5 and 6 (see Supplementary Material) 
The different capillary models that were put forward to ture dependence of the chemical potential $\mu=\gamma \Omega \kappa$, where $\Omega$ is an atomic volume and $\kappa=\kappa_{\|}+\kappa_{\perp}$ the sum of the curvature components parallel and perpendicular to the surface 22. It is important to notice that $\kappa$ is negative metal. The gradient of chemical potential $\Delta \mu$ between a point along the front and a point far away on the pristine film is often used to estimate the flux of diffusing material from or to the dewetting front. Initially, Brandon and

${ }_{475}$ Bradshaw 6] modelled the propagation of a rim of height $r$ higher than the pristine film thickness $h$ by considering $\kappa_{\|} \ll \kappa_{\perp}$ and $\kappa=\kappa_{\perp}=1 / r$ (see Fig. 12 a, side view A), so that $\Delta \mu=\Omega \gamma / r$. However, later on, Jiran and Thompson [14] observed that the propagation occurred where the

${ }_{480}$ rim was the thinnest (see Fig. 12 a, side view B). They suggested that the dewetting material was accumulated on the sides of a propagating finger with no rim in the direction of propagation. Within their model, $\kappa_{\perp}=1 / h$ and $\kappa_{\|}=-1 / r^{\prime}$ where $r^{\prime}$ is the radius of the tip; the negative

485 sign results from the concavity is outside of the material. Therefore, they wrote the chemical potential gradient as:

$$
\Delta \mu=\Omega \gamma\left(-\frac{1}{r^{\prime}}+\frac{1}{h}\right)
$$

and within the restrictive assumption $r^{\prime} \gg h$, they ob- ${ }^{520}$ tained a constant $\Delta \mu=\gamma \Omega / h$ in agreement with their kinetics observations.

Yet our findings are in contradiction with this model. To illustrate this discrepancy, two points A and B are highlighted in Fig. 12 a. Accordingly to our image analysis of in-plane curvature, the highest propagation speed in vacuum is observed for $r^{\prime} \simeq h$ (see Fig. 11-bottom) in other terms at the saddle point B of Fig. 12 a. Here the effects of both in-plane and out-of-plane curvatures will compensate each other $\left(\kappa_{B} \simeq 0\right)$. Yet, according to Eq. 3. $\mathrm{a}^{530}$ much faster propagation should be expected at point $\mathrm{A}$ where both curvatures add leading to $\kappa_{A}>0$. In summary, while our experimental findings about the shape of the front match the observations of Ref. [14, the capillary model based on the assumption $r^{\prime} \gg h$ is inadequate to ${ }^{53}$ explain a faster propagation at point $B$.

Recently, Zucker et al. 37. developed a new approach to describe corner instabilities observed during the dewetting of a single crystal film. It consists in a prompt retraction of the film where a substantial in-plane negative ${ }^{540}$ curvature is observed. However, in the corner instability model, the derivative of the retraction speed at the corner with respect to the in-plane radius of curvature $b_{t i p}$ is always positive. This is the case independently on the out-of-plane curvature. If we extended this model to $\mathrm{a}^{545}$ polycrystalline film, it would fail describing the increase of propagation rate with the increasing in-plane negative curvature (decrease of $b_{\text {tip }}$ ). Therefore, corner instability cannot explain our observations.

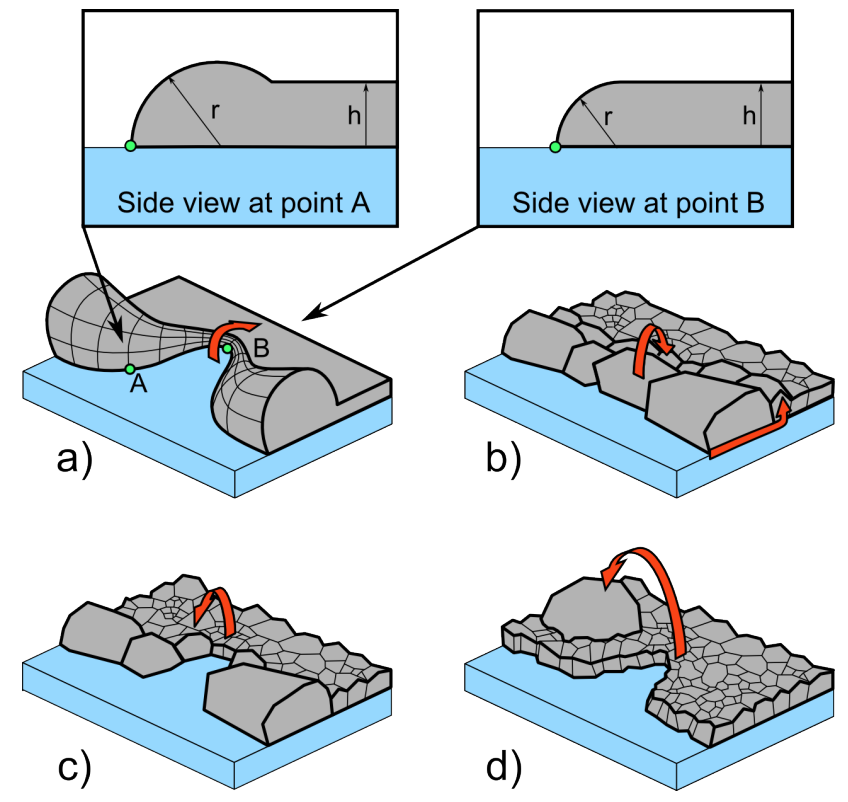

Figure 12: Different suggested dewetting mechanisms of polycrystalline films. a) Ref. 14, b) Refs. 16 4 with surface or interface transport, our model c) in vacuum d) and in oxygen-rich atmosphere.

At this point we conclude that "macroscopic" surface curvature (i.e. at the scale of the layer thickness) is not the driving force of dewetting in our system. We are now discussing other possibilities.

In parallel works [17, 16, 4] based on post mortem observations, the arguments of chemical potential and curvature of the front were also challenged. Instead of using capillary concepts, the explanations were based on grain growth and shrinkage (see Fig. 12p). The study of Kovalenko et al. [17] and silver dewetting in oxygen atmosphere as compared in Ref. [13. concluded to the lack of propagating rim. In contrast, silver dewetting in vacuum appears to be more similar to the dewetting observed in the other two studies [16, 4,. There, the retracting rim was described as the successive growth and shrinkage of grains surrounding the holes. This mechanism is supposed to ensure hole propagation. Atiya et al. 4 describe surface diffusion to be the main pathway, whereas Kosinova et al. [16] hold interface diffusion and grain boundary sliding as main transport mechanism.

Our SEM experiments confirm that grain shrinkage is ensuring hole propagation, in agreement with these models. However, it does not consist in the propagation of a rim. Once grains have grown, they will not shrink during the propagation. The grains that shrink are the smallest ones and are present where the front height is comparable with the initial thickness of the layer. This is particularly visible on the sequence of images in Fig. 6. These findings are in line with Jiran's observations [14 but with different morphologies in vacuum or in oxygen.

It is possible to explain the differences of dewetting morphologies obtained in both annealing atmospheres based on grain selection only. In vacuum, many grains grow, 
leaving less free space for hole propagation. The regions were the grains are the smallest are situated where the 605 in plane curvature of the holes is the most negative (see Fig. 12. and Fig. 6), leading to fingering and propagation at the tips. In oxygen, only a few grains are selected to grow (see [13]) leaving far more possibilities for the expanding holes. Moreover, the selected grains can grow as610 far as $1 \mu \mathrm{m}$ away from the dewetting front (see Fig. $12 \mathrm{~d}$ and Fig. 57. The shape of the holes is far less constrained an in vacuum.

However, why grain growth is so dependent on the annealing atmosphere remains unanswered and will be in-615 vestigated in future studies. Following factors can be put forward to explain grain selection:

- the change of the diffusion coefficient 31 itself should not be the limiting factor. Yet in line with diffusion enhancement in oxygen, Yoshihara et al. 35. claims that oxygen adsorption prevent silver adatoms from ${ }^{620}$ refilling vacancies or nucleating new facets. If oxygen coverage is less pronounced on some facets, this could imply a higher growth rate at specific places.

- the modification of the hierarchy of facet surface energies leading to different facet selection depending on the atmosphere.

- the local configuration of the grains is also expected to play its role in the grain selection, as it was suggested in previous works [27]. Yet this local configuration is also influenced by the atmosphere in terms ${ }_{635}$ of surface energy.

\section{Conclusion}

The dynamics of silver solid-state dewetting has been monitored thanks to in situ HT-ESEM and complementary TEM and AFM experiments. To study the evolution of the holes based on their in-plane curvature, a new pro-645 cedure of image processing was implemented to determine the local speed of the whole dewetting front given its local curvature.

Hole propagation does not involve any rim movement.650 Instead, propagation occurs only on the parts of the layer that still have the same thickness as the pristine film. While this observation is in agreement with the work of Jiran and Thompson 14 on polycrystalline films, the cap-655 illary models based on curvature fail to explain our results, even on amorphous substrate. Instead, grains by themselves seem to play a major role: surface coverage decreases only due to the disappearance of the smaller grains ${ }^{660}$ located on the dewetting front that shrink in favour of selected ones.

It was found that the oxygen in the annealing atmo600 sphere has a dramatic effect on the dewetting morphology ${ }^{665}$ of silver. During induction, grains drastically reorganizes but oxygen impacts this evolution by facilitating the creation of holes, probably by lowering the surface energy of silver and favouring grain boundary grooving. Then, during hole propagation, oxygen increases the kinetics of the process. By narrowing the selection of growing grain, oxygen leaves more possibilities for the holes to expand. While the origin of the selection of grains remains to be understood, the nature of the dewetting atmosphere is a key parameter to consider to achieve a good control of designed silver structures through templated dewetting. The size and the shape of the silver structures obtained are clearly dependent on the oxygen content in the atmosphere, an experimental parameter that can be tuned to reach a desired morphology.

Acknowledgements. We gratefully thank Hervé Montigaud from SVI and Julie Bourgon from Institut de Chimie des Matériaux de Paris Est for the ACOM studies of the dewetting.

[1] P. M. Agrawal, B. M. Rice, and D. L. Thompson. Predicting trends in rate parameters for self-diffusion on FCC metal surfaces. Surf. Sci., 515:21-35, 2002.

[2] D. Amram, L. Klinger, N. Gazit, H. Gluska, and E. Rabkin. Grain boundary grooving in thin films revisited: The role of interface diffusion. Acta Mater., 69:386-396, May 2014.

[3] D. Amram, L. Klinger, and E. Rabkin. Anisotropic hole growth during solid-state dewetting of single-crystal AuFe thin films. Acta Mater., 60(6-7):3047-3056, Apr. 2012.

[4] G. Atiya, D. Chatain, V. Mikhelashvili, G. Eisenstein, and W. D. Kaplan. The role of abnormal grain growth on solidstate dewetting kinetics. Acta Mater., 81:304-314, Dec. 2014.

[5] A. Benkouider, A. Ronda, T. David, L. Favre, M. Abbarchi, M. Naffouti, J. Osmond, a. Delobbe, P. Sudraud, and I. Berbezier. Ordered arrays of Au catalysts by FIB assisted heterogeneous dewetting. Nanotech., 26(50):505602, Dec. 2015.

[6] R. Brandon and F. J. Bradshaw. The Mobility of the surface atoms of copper and silver evaporated deposits. Royal Aircraft Establishment, Tech. Rep., (66093), 1966.

[7] F. H. Buttner, R. Funk, and H. Udin. ADSORPTION OF OXYGEN ON SILVER. J. Phys. Chem., 56(1):657-660, 1952.

[8] D. Chatain, F. Chabert, and V. Ghetta. New Experimental Setup for Wettability Characterization under Monitored Oxygen Activity: II, Wettability of Sapphire by Silver-Oxygen Melts. J. Am. Ceram. Soc, 77(1):197-201, 1994.

[9] V. Derkach, A. Novick-Cohen, A. Vilenkin, and E. Rabkin. Grain boundary migration and grooving in thin 3-D systems. Acta Mater., 65(2014):194-206, Feb. 2014.

[10] J. Fiala and J. Ćdek. Surface and grain boundary energies of silver at oxygen pressures lower than $10 \mathrm{~Pa}$. Philos.l Mag., 32(1):251, 1075.

[11] M. Inman and H. Tipler. Interfacial energy and composition in metal alloys. Met. R., 8, 1963.

[12] P. Jacquet, M. Kildemo, J. Teisseire, I. Gozhyk, J. Jupille, and R. Lazzari. Monitoring silver solid-state dewetting with in situ ellipsometry. App. Surf. Sci., Oct. 2016.

[13] P. Jacquet, R. Podor, J. Ravaux, J. Teisseire, I. Gozhyk, J. Jupille, and R. Lazzari. Grain growth: The key to understand solid-state dewetting of silver thin films. Scripta Mater., 115:128-132, Apr. 2016.

[14] E. Jiran and C. V. Thompson. Capillary Instabilities in Thin Films films. J. Elec. Mat., 19(11):1153, 1990.

[15] A. Kosinova, L. Klinger, O. Kovalenko, and E. Rabkin. The role of grain boundary sliding in solid-state dewetting of thin polycrystalline films. Scripta Mater., 82:33-36, 2014.

[16] A. Kosinova, O. Kovalenko, L. Klinger, and E. Rabkin. Mechanisms of solid-state dewetting of thin Au films in different annealing atmospheres. Acta Mater., 83:91-101, 2015.

[17] O. Kovalenko, J. Greer, and E. Rabkin. Solid-state dewetting 
of thin iron films on sapphire substrates controlled by grain 740 boundary diffusion. Acta Mater., 61(9):3148-3156, May 2013.

[18] J. Kudrman. RELATIVE GRAIN BOUNDARY FREE ENERGY. Czech. J. Phys. B, 19:1337-1342, 1969.

[19] J. Y. Kwon, T. S. Yoon, K. B. Kim, and S. H. Min. Comparison of the agglomeration behavior of $\mathrm{Au}$ and $\mathrm{Cu}$ films sputter deposited on silicon dioxide. J. App. Phy., 93(6):3270, 2003.

[20] F. Leroy, . Borowik, F. Cheynis, Y. Almadori, S. Curiotto, M. Trautmann, J. Barbe, and P. Müller. How to control solid ${ }^{74}$ state dewetting: A short review. Surf. Sci. Rep., 71(2):391-409, Apr. 2016.

[21] L. M. Molina, S. Lee, K. Sell, G. Barcaro, A. Fortunelli, B. Lee, S. Seifert, R. E. Winans, J. W. Elam, M. J. Pellin, I. Barke, V. V. Oeynhausen, Y. Lei, R. J. Meyer, J. A. Alonso, A. Fraile, A. Kleibert, S. Giorgio, C. R. Henry, K.-h. Meiwes-broer, and750 S. Vajda. Size-dependent selectivity and activity of silver nanoclusters in the partial oxidation of propylene to propylene oxide and acrolein : A joint experimental and theoretical study. Cat. Today, 160(2011):116-130, 2010.

[22] W. W. Mullins. Theory of Thermal Grooving. J. App. Phy., 28(3):333, 1957

[23] W. W. Mullins. The statistical self-similarity hypothesis in grain growth and particle coarsening. J. App. Phy., 59(4):1341, 1986.

[24] A. J. Nagy, G. Mestl, D. Herein, G. Weinberg, E. Kitzelmann, and R. Schlögl. The Correlation of Subsurface Oxygen Diffusion with Variations of Silver Morphology in the Silver Oxygen System. J. Catalysis, 182:417-429, 1999.

[25] F. Niekiel, S. M. Kraschewski, P. Schweizer, B. Butz, and E. Spiecker. Texture evolution and microstructural changes during solid-state dewetting: A correlative study by complementary in situ TEM techniques. Acta Mater., 115(2016):230-241, Aug. 2016.

[26] F. Niekiel, P. Schweizer, S. M. Kraschewski, B. Butz, and E. Spiecker. The process of solid-state dewetting of Au thin films studied by in situ scanning transmission electron microscopy. Acta Mater., 90:118-132, May 2015.

[27] W. B. Pennebaker. Hillock Growth and Stress Relief in Sputtered Au Films. J. App. Phy., 40(1):394-400, Jan. 1969.

[28] R. Podor, D. Pailhon, J. Ravaux, and H.-P. Brau. Development of an Integrated Thermocouple for the Accurate Sample Temperature Measurement During High Temperature Environmental Scanning Electron Microscopy (HT-ESEM) Experiments. Microscopy and microanalysis, 21(2):307-312, Apr. 2015.

[29] A. E. B. Presland, G. L. Price, D. L. Trimm, and T. Heath. Hillock formation by surface diffusion on thin silver films. Surf. Sci., 29:424-434, 1972.

[30] E. F. Rauch, J. Portillo, S. Nicolopoulos, D. Bultreys, S. Rouvimov, and P. Moeck. Automated Nanocrystal Orientation and Phase Mapping in the Transmission Electron Microscope on the Basis of Precession Electron Diffraction. Z. Kistallogr., 225:103109, 2010 .

[31] G. E. Rhead. Surface self-diffusion of silver in various atmospheres. Acta Metall., 13:223, 1965.

[32] G. Schmidl, J. Dellith, H. Schneidewind, D. Zopf, O. Stranik, A. Gawlik, S. Anders, V. Tympel, C. Katzer, F. Schmidl, and W. Fritzsche. Formation and characterization of silver nanoparticles embedded in optical transparent materials for plasmonic sensor surfaces. Mater. Sci. Eng. B, 193:207-216, 2015.

[33] S. Stankic, R. Cortes-Huerto, N. Crivat, D. Demaille, J. Goniakowski, and J. Jupille. Equilibrium shapes of supported silver clusters. Nanoscale, 5(6):2448-53, Mar. 2013.

[34] C. V. Thompson. Solid State Dewetting of Thin Films. Annu. Rev. Matter., 42:399, 2012.

[35] K. Yoshihara and K. Nii. The effect of Oxygen Potential on the Surface Self diffusion Coefficient of Silver. Trans. J. I. Metall., 20:533, 1979.

735 [36] R. V. Zucker, G. H. Kim, W. Craig Carter, and C. V. Thompson. A model for solid-state dewetting of a fully-faceted thin film. C. R. Phy., 14(7):564-577, Aug. 2013.

[37] R. V. Zucker, G. H. Kim, J. Ye, W. C. Carter, and C. V. Thompson. The mechanism of corner instabilities in single-crystal thin films during dewetting. J. App. Phy., 119(12):125306, Mar. 2016.

Supplementary Materials. SEM movies of the dewetting under vacuum and oxygen at different magnifications.

video 1: low magnification, $40 \mathrm{~nm}$ in vacuum, $350^{\circ} \mathrm{C}$

video 2: low magnification, $40 \mathrm{~nm}$ in oxygen (400 $\mathrm{Pa})$, $350^{\circ} \mathrm{C}$

video 3: high magnification, $80 \mathrm{~nm}$ in oxygen (400 Pa), $480^{\circ} \mathrm{C}$

video 4: high magnification, $80 \mathrm{~nm}$ in vacuum, $570^{\circ} \mathrm{C}$

video 5: medium magnification, $60 \mathrm{~nm}$ in oxygen (400 $\mathrm{Pa})$, $270^{\circ} \mathrm{C}$

video 6: medium magnification, $60 \mathrm{~nm}$ in vacuum, $430^{\circ} \mathrm{C}$ 